\title{
Clinical utility gene card for: Progressive familial intrahepatic cholestasis type 2
}

\author{
Emmanuel Gonzales ${ }^{1,2}$, Anne Spraul ${ }^{3}$ and Emmanuel Jacquemin ${ }^{\star, 1,2}$ \\ European Journal of Human Genetics (2014) 22, doi:10.1038/ejhg.2013.187; published online 28 August 2013
}

\section{DISEASE CHARACTERISTICS}

1.1 Name of the disease (synonyms)

1. Progressive familial intrahepatic cholestasis type 2 (PFIC2).

2. Bile salt export pump (BSEP) deficiency.

3. Initially reported under the name Byler syndrome.

Byler syndrome refers to normal gamma-glutamyltransferase (GGT) level chronic intrahepatic cholestasis observed in children usually during the first year of life. ${ }^{1}$ Later, PFIC1 (Byler disease) ${ }^{2}$ and $\mathrm{PFIC}^{3}$ were identified. The terms PFIC2 or BSEP deficiency should be used preferentially.

\subsection{OMIM\# of the disease}

601847.

1.3 Name of the analysed genes or DNA/chromosome segments ATP-binding cassette sub-family B or ABCB11.

Chromosome 2q24.3-2q31.1 (g.169487695-169596079).

\subsection{OMIM\# of the gene(s)}

603201.

\subsection{Mutational spectrum}

More than 100 mutations have been reported, mostly point mutations (missense, nonsense and splicing) located throughout the gene's 27 exons, but also small deletions, insertions and duplications. ${ }^{4-8}$

Mutations of $A B C B 11$ can be found in the NHLBI ESP Exome Variant Server database (http://evs.gs.washington.edu/EVS/).

\subsection{Analytical methods}

Bidirectional $A B C B 11$ gene sequencing of coding exons and their flanking intronic junctions (RefSeq accession number NM_003742.2).

As BSEP expression is virtually restricted to hepatocytes, transcripts of $A B C B 11$ can only be studied on liver biopsy.

When the analysis fails to identify both mutant alleles, a search for (partial) gene deletion or duplication by multiplex ligation-dependant probe identification may be considered.

\subsection{Analytical validation}

Independent sequencing of both strands of DNA (forward and reverse).
When heterozygosity for two mutations is found, testing of the patient's parents is recommended to confirm that the defect is biallelic.

In case of newly identified variation, a polymorphism has to be excluded by testing a set of at least 100 control chromosomes of the same ethnic origin. Pathogenicity of such novel variations has to be tested by in silico prediction methods. Moreover, protein stability and trafficking may be studied in cell lines on a research basis. Concerning splice site variants, their pathogenic nature should be studied by cDNA analysis.

\subsection{Estimated frequency of the disease}

(Incidence at birth ('birth prevalence') or population prevalence. If known to be variable between ethnic groups, please report):

The population prevalence of PFIC2 is unknown.

However, it is estimated around 1/100 000 births.

\subsection{Diagnostic setting}

\begin{tabular}{lll}
\hline & Yes & No \\
\hline A. (Differential) diagnostics & $\bigotimes$ & $\square$ \\
B. Predictive testing & $\square$ & $\square$ \\
C. Risk assessment in relatives & $\bigotimes$ & $\square$ \\
D. Prenatal & $\bigotimes$ & $\square$ \\
\hline
\end{tabular}

\section{Comment:}

PFIC2 is due to defects in the $A B C B 11$ gene, encoding the BSEP protein, resulting in impaired biliary bile acid secretion, which leads to decreased bile flow and bile salt accumulation in hepatocytes with ongoing severe hepatocellular damage. Transmission is autosomal recessive. PFIC2 should be suspected in children with a clinical history of cholestasis of unknown origin after exclusion of other main causes of cholestasis presenting with normal serum GGT activity and high serum bile acid concentration. ${ }^{1}$ Usually, serum $\alpha$-fetoprotein level is elevated and alanine aminotransferase values are over fivefold the upper limit of normal. ${ }^{7}$ Liver ultrasonography is usually normal but may reveal a huge gallbladder and sometimes biliary stones. Microscopy of the liver reveals canalicular and hepatocellular cholestasis, the absence of true ductular proliferation with only periportal biliary metaplasia of hepatocytes, pronounced lobular and portal fibrosis and inflammation, hepatocellular necrosis and

\footnotetext{
1INSERM, UMR-S757, University of Paris-Sud 11, Orsay, France; ${ }^{2}$ Pediatric Hepatology and Liver transplantation Unit, National Reference Centre for Rare Pediatric Liver Diseases, Bicêtre Universitary Hospital, Faculty of Medicine Paris-Sud, University of Paris-Sud 11, Assistance Publique-Hôpitaux de Paris, Le Kremlin-Bicêtre, France; ${ }^{3}$ Biochemistry Unit, Bicêtre Universitary Hospital, Faculty of Medicine Paris-Sud, University of Paris-Sud 11, Assistance Publique-Hôpitaux de Paris, Le Kremlin-Bicêtre, France *Correspondence: Professor E Jacquemin, Service d'hépatologie et de transplantation hépatique pédiatriques, CHU Bicêtre, 77 rue du Général Leclerc, Le Kremlin-Bicêtre 94275, France. Tel: +33 145213164; Fax: +33 145212816; E-mail: emmanuel.jacquemin@bct.aphp.fr
} 
giant cell transformation. BSEP immunostaining helps for diagnosis as it is usually negative in patients with PFIC2.5,7,9 When performed, cholangiography shows a normal biliary tree and allows bile collection. Biliary lipid analysis reveals dramatically decreased biliary bile salt concentration. Genotyping confirms the diagnosis. In the setting of neonatal cholestasis with normal serum GGT activity, normal serum level of bile acid suggests primary bile acid synthesis defects. When bile acid concentrations in serum are elevated in cholestasis with normal serum GGT activity of early infancy, the two main differential diagnostic considerations are PFIC1 and liver disease related to mitochondrial respiratory chain disorders.

Some patients with BSEP deficiency present with benign recurrent intrahepatic cholestasis type 2 (BRIC2, OMIM 605479). ${ }^{10}$ They suffer from bouts of cholestasis and liver pathological examination, when performed, shows only minimal fibrosis. In some patients, BSEP deficiency initially presents as BRIC2 and an evolution towards a PFIC2 phenotype is observed illustrating that BSEP deficiency represents a phenotypic continuum from BRIC2 to PFIC2. ${ }^{7}$ Life-long specialised medical follow-up is mandatory.

Monoallelic mutation of $A B C B 11$, predisposes to drug-induced cholestasis (DIC), intrahepatic cholestasis of pregnancy type $2,11,12$ transient neonatal cholestasis ${ }^{13}$ and biliary lithiasis. ${ }^{7}$ Specialised medical follow-up should also be offered to heterozygous patients (mainly those who are symptomatic).

\section{TEST CHARACTERISTICS}

\begin{tabular}{|c|c|c|c|c|}
\hline & \multicolumn{2}{|c|}{ Genotype or disease } & \multirow{2}{*}{$\begin{array}{l}\text { A: True positives } \\
\text { B: False positives }\end{array}$} & \multirow{2}{*}{$\begin{array}{l}\text { C: False negative } \\
\text { D: True negative }\end{array}$} \\
\hline & Present & Absent & & \\
\hline \multicolumn{5}{|l|}{ Test } \\
\hline \multirow[t]{2}{*}{ Positive } & A & B & Sensitivity: & $A /(A+C)$ \\
\hline & & & Specificity: & $D /(D+B)$ \\
\hline \multirow[t]{2}{*}{ Negative } & C & D & Positive predictive value: & $A /(A+B)$ \\
\hline & & & Negative predictive value: & $\mathrm{D} /(\mathrm{C}+\mathrm{D})$ \\
\hline
\end{tabular}

\subsection{Analytical sensitivity}

(proportion of positive tests if the genotype is present)

Approximately $100 \%$.

\subsection{Analytical specificity}

(proportion of negative tests if the genotype is not present)

Approximately 100\%.

\subsection{Clinical sensitivity}

\section{(proportion of positive tests if the disease is present)}

The clinical sensitivity can be dependent on variable factors such as age or family history. In such cases, a general statement should be given, even if a quantification can only be made case by case.

Approximately 90\%.

In few patients with a PFIC2 phenotype (including a negative BSEP immunostaining on liver biopsy), none or only one disease-causing mutation has been identified. ${ }^{5-8}$

\subsection{Clinical specificity}

(proportion of negative tests if the disease is not present)

The clinical specificity can be dependent on variable factors such as age or family history. In such cases, a general statement should be given, even if a quantification can only be made case by case.

Approximately 100\%.

\subsection{Positive clinical predictive value}

(life-time risk to develop the disease if the test is positive)

There is no case report of an asymptomatic person harbouring two predictedly pathogenic mutations in biallelic state. In case of known disease-causing mutations, virtually $100 \%$ of affected individuals develop cholestasis. However, PFIC2 constitute the severe side of the clinical spectrum of BSEP deficiency and some patients may suffer from BRIC2. Evolution of BRIC2 patients towards a PFIC2 phenotype has been reported. ${ }^{7}$ In approximately half patients with PFIC2, liver transplantation is required before adulthood.

\subsection{Negative clinical predictive value}

(probability not to develop the disease if the test is negative) Assume an increased risk based on family history for a non-affected person. Allelic and locus heterogeneity may need to be considered.

Index case in that family had been tested:

Practically $100 \%$.

Index case in that family had not been tested:

Practically $100 \%$.

\section{CLINICAL UTILITY}

3.1 (Differential) diagnostics: the tested person is clinically affected

(To be answered if in 1.9 'A' was marked)

\subsubsection{Can a diagnosis be made other than through a genetic test?}

\begin{tabular}{|c|c|c|}
\hline No & $\square$ (Continue with 3.1.4) & \\
\hline \multirow[t]{7}{*}{ Yes } & $\nabla$ & \\
\hline & Clinically & $\square$ \\
\hline & Imaging & $\square$ \\
\hline & Endoscopy & $\square$ \\
\hline & Biochemistry & $\square$ \\
\hline & Electrophysiology & $\square$ \\
\hline & Other (please describe) & \\
\hline
\end{tabular}

\subsubsection{Describe the burden of alternative diagnostic methods to the} patient

A combined clinical, biochemical, radiological and histological approach associated with liver immunostaining and biliary lipid analysis can allow a PFIC2 diagnosis in most cases. However, invasive procedures, which can only be performed in few centers with a high level of expertise - such as liver biopsy and bile collection by endoscopic retrograde cholangiopancreatography or by direct vesicular puncture - are required. The use of genetic study to confirm clinicopathologic diagnoses - made through study of clinical, biochemical, imaging-study and histopathologic findings, with immunostaining and ultrastrucuture evaluation - is generally prudent.

3.1.3 How is the cost effectiveness of alternative diagnostic methods to be judged?

Unknown. 
3.1.4 Will disease management be influenced by the result of a genetic test?

No $\square$

Yes $\otimes$

Therapy Ursodeoxycholic acid (UDCA) therapy should be initiated in all (please patients to prevent liver damage, but is not fully efficient. ${ }^{7,14}$

describe) Rifampicin is helpful to control pruritus. In some patients, biliary diversion may decrease cholestasis. ${ }^{15}$ Nasobiliary drainage may help to select potential responders to biliary diversion. So far, clear genotype-phenotype correlation data are missing and remain to be defined in order to identify those PFIC2 patients who could benefit from UDCA or biliary diversion. Preliminary data suggest that PFIC2 patients with some specific missense mutations may respond well to biliary diversion ${ }^{16}$ and/or UDCA therapy. ${ }^{7}$ In vitro studies showed that some missense BSEP mutations affect protein processing/ trafficking causing retention in the endoplasmic reticulum and subsequent endoplasmic reticulum-associated degradation. Several drugs able to partially correct BSEP mutants trafficking have been identified in vitro. These results led to new therapeutic approach strategy such as tailored mutationspecific pharmacotherapy. ${ }^{17}$ In the future, therapies such as cell, gene or specific targeted pharmacological therapies (eg, FXR inducers, chaperone drugs) might represent an alternative therapy for all types of PFIC. Successful mutationspecific chaperone therapy with 4-phenylbutyrate was recently reported in a child with PFIC2. ${ }^{18}$

Prognosis Genetic characterisation of the patients is required for (please genotype-phenotype correlation analysis. However, because of describe) severe cholestasis, liver failure or hepatocellular carcinoma, half of patients are ultimately candidates for liver transplantation. ${ }^{7}$ After liver transplantation a recurrence of PFIC in the allograft because of allo-immunisation of the recipient against BSEP protein of the donor has been reported. ${ }^{19,20}$

Management Close monitoring of hepatocellular carcinoma should be offered (please from the first year of life. Life-long specialised medical followdescribe) up is mandatory.

3.2 Predictive setting: the tested person is clinically unaffected but carries an increased risk based on family history

(To be answered if in 1.9 'B' was marked).

3.2.1 Will the result of a genetic test influence lifestyle and prevention? If the test result is positive (please describe).

If the test result is negative (please describe).

3.2.2 Which options in view of lifestyle and prevention does a person at-risk have if no genetic test has been done (please describe)?

Not applicable.

3.3 Genetic risk assessment in family members of a diseased person (To be answered if in 1.9 ' $C$ ' was marked).

3.3.1 Does the result of a genetic test resolve the genetic situation in that family?

If the two disease-causing mutations have been identified in the diseased person, family members with a negative test are not at risk for being affected.

3.3.2 Can a genetic test in the index patient save genetic or other tests in family members?
Yes: in case of a known familial mutation, carriership can be confirmed or excluded.

3.3.3 Does a positive genetic test result in the index patient enable a predictive test in a family member?

As BSEP deficiency at a heterozygous status predisposes to develop intrahepatic cholestasis of pregnancy type 2, drug-induced cholestasis, transient neonatal cholestasis and also biliary lithiasis, a predictive test can be offered to family members.

In asymptomatic relatives with monoallelic mutation, information regarding the particular risks should be given. Specialised medical care (including UDCA treatment) and follow-up should be offered to symptomatic relatives with monoallelic mutation.

\subsection{Prenatal diagnosis}

(To be answered if in 1.9 'D' was marked).

\subsubsection{Does a positive genetic test result in the index patient enable a} prenatal diagnosis?

Yes, prenatal diagnosis can be proposed if a disease-causing mutation has been identified in each parent.

\section{IF APPLICABLE, FURTHER CONSEQUENCES OF TESTING}

Please assume that the result of a genetic test has no immediate medical consequences. Is there any evidence that a genetic test is nevertheless useful for the patient or his/her relatives? (Please describe).

Genetic counseling is always useful for the family.

Review of the analytical and clinical validity as well as of the clinical utility of DNA based.

Testing for mutations in the $A B C B 11$ gene in diagnostic, predictive and prenatal settings and for risk assessment in relatives.

\section{CONFLICT OF INTEREST}

The authors declare no conflict of interest.

\section{ACKNOWLEDGEMENTS}

This work was supported by EuroGentest2 (Unit 2: 'Genetic testing as part of health care'), a Coordination Action under FP7 (Grant Agreement Number 261469) and the European Society of Human Genetics.

1 Davit-Spraul A, Gonzales E, Baussan C et al: Progressive familial intrahepatic cholestasis. Orphanet J Rare Dis 2009; 4: 1.

2 Bull L, Eijk MJ, Pawlikowska L et al: A gene encoding a P-type ATPase mutated in two forms of hereditary cholestasis. Nat Genet 1998; 18: 219-224.

3 Strautnieks SS, Bull LN, Knisely AS et al: A gene encoding a liver-specific ABC transporter is mutated in progressive familial intrahepatic cholestasis. Nat Genet 1998; 20: 233-238.

4 Jansen PLM, Strautnieks SS, Jacquemin E et al: Hepatocanalicular bile salt export pump deficiency in patients with progressive familial intrahepatic cholestasis. Gastroenterology 1999; 117: 1370-1379.

5 Strautnieks SS, Byrne JA, Pawlikowska L et al: Severe bile salt export pump deficiency: 82 different ABCB11 mutations in 109 families. Gastroenterology 2008; 134: 1203-1214.

6 Knisely AS, Strautnieks SS, Meier Y et al: Hepatocellular carcinoma in ten children under five years of age with bile salt export pump deficiency. Hepatology 2006; 44: 478-486.

7 Davit-Spraul A, Fabre M, Branchereau S et al: ATP8B1 and ABCB11 analysis in 62 children with normal GGT PFIC: phenotypic differences between PFIC1 and PFIC2 and natural history. Hepatology 2010; 51: 1645-1655.

8 Pawlikowska L, Strautnieks S, Jankowska I et al: Differences in presentation and progression between severe FIC1 and BSEP deficiencies. J Hepatol 2010; 53: 170-178.

9 Evason K, Bove KE, Finegold MJ et al: Morphologic findings in progressive familial intrahepatic cholestasis 2 (PFIC2): correlation with genetic and immunohistochemical studies. Am J Surg Pathol 2011; 35: 687-696. 
10 van Mil SW, van der Woerd WL, van der Brugge G et al: Benign recurrent intrahepatic cholestasis type 2 is caused by mutations in ABCB11. Gastroenterology 2004; 127: 379-384.

11 Pauli-Magnus C, Lang T, Meier $\mathrm{Y}$ et al: Sequence analysis of bile salt export pump (ABCB11) and multidrug resistance p-glycoprotein 3 (ABCB4, MDR3) in patients with intrahepatic cholestasis of pregnancy. Pharmacogenetics 2004; 14 91-102.

12 Pauli-Magnus C, Meier PJ, Stieger B: Genetic determinants of drug-induced cholestasis and intrahepatic cholestasis of pregnancy. Semin Liver Dis 2010; 30: 147-159.

13 Hermeziu B, Sanlaville D, Girard M et al: Heterozygous bile salt export pump deficiency: a possible genetic predisposition to transient neonatal cholestasis. J Pediatr Gastroenterol Nutr 2006; 42: 7-8.

14 Jacquemin E, Hermans D, Myara A et al: Ursodeoxycholic acid therapy in pediatric patients with progressive familial intrahepatic cholestasis. Hepatology 1997; 25: 519-523.
15 Stapelbroek JM, van Erpecum KJ, Klomp LW et al: Liver disease associated with canalicular transport defects: current and future therapies. J Hepatol 2010; 52: 258-271.

16 Balistreri WF, Bezzera JA, Jansen P et al: Intrahepatic cholestasis: summary of an American association for study of liver diseases single-topic conference. Hepatology 2005; 42: 222-235.

17 Gonzales E, Jacquemin E: Mutation specific drug therapy for progressive familial or benign recurrent intrahepatic cholestasis: a new tool in a near future? J Hepatol 2010; 53: 385-387.

18 Gonzales E, Grosse B, Cassio D et al: Successful mutation-specific chaperone therapy with 4-phenylbutyrate in a child with progressive familial intrahepatic cholestasis type 2. J Hepatol 2012; 57: 695-698.

19 Keitel V, Burdelski M, Vojnisek Z et al: De novo bile salt transporter antibodies as a possible cause of recurrent graft failure after liver transplantation: a novel mechanism of cholestasis. Hepatology 2009; 50: 510-517.

20 Jara $\mathrm{P}$, Hierro L, Martínez-Fernández $\mathrm{P}$ et al: Recurrence of bile salt export pump deficiency after liver transplantation. N Engl J Med 2009; 361: 1359-1367. 